\title{
Wave fields in double-slit experiments with wave-driven droplets
}

Rode, Mads Boje; Madsen, Jacob; Andersen, Anders

Published in:

Physical Review Fluids

Link to article, DOI:

10.1103/PhysRevFluids.4.104801

Publication date:

2019

Document Version

Publisher's PDF, also known as Version of record

Link back to DTU Orbit

Citation (APA):

Rode, M. B., Madsen, J., \& Andersen, A. (2019). Wave fields in double-slit experiments with wave-driven droplets. Physical Review Fluids, 4(10), [104801]. https://doi.org/10.1103/PhysRevFluids.4.104801

\section{General rights}

Copyright and moral rights for the publications made accessible in the public portal are retained by the authors and/or other copyright owners and it is a condition of accessing publications that users recognise and abide by the legal requirements associated with these rights.

- Users may download and print one copy of any publication from the public portal for the purpose of private study or research.

- You may not further distribute the material or use it for any profit-making activity or commercial gain

- You may freely distribute the URL identifying the publication in the public portal

If you believe that this document breaches copyright please contact us providing details, and we will remove access to the work immediately and investigate your claim 


\title{
Wave fields in double-slit experiments with wave-driven droplets
}

\author{
Mads Rode, Jacob Madsen, ${ }^{*}$ and Anders Andersen ${ }^{\dagger}$ \\ Department of Physics, Technical University of Denmark, DK-2800 Kgs. Lyngby, Denmark
}

(Received 16 March 2017; published 17 October 2019)

\begin{abstract}
We present an experimental study of surface wave fields in single- and double-slit experiments with single wave-driven droplets of silicone oil that bounce on a vertically vibrated bath of the same liquid. During slit passage, we observe significant reduction of the central wave crest behind the droplet and disruption of the horseshoe-shaped wave field that is characteristic of a droplet over flat topography. In the double-slit experiments, we find a weak wave field in and through the slit that is not visited by the droplet, and there are only minute differences between the single- and double-slit experiments in the wave field along the center line of the slit that is visited by the droplet. We discuss our observations in relation to the slit experiments by Couder and Fort [Phys. Rev. Lett. 97, 154101 (2006)] and Pucci et al. [J. Fluid Mech. 835, 1136 (2018)].
\end{abstract}

DOI: 10.1103/PhysRevFluids.4.104801

\section{INTRODUCTION}

A millimetric droplet of silicone oil can bounce indefinitely on the surface of a vertically vibrated bath of the same liquid [1]. Surprisingly, at vibration amplitudes just below the threshold for Faraday surface waves, a droplet can self-propel horizontally over the surface due to the waves that are generated as it bounces [2]. Such wave-driven droplets have recently been the subject of intense studies to explore both their fascinating fluid dynamics [3-14] and their thought-provoking relation to quantum mechanics [15-30]. The exploration of the relation to quantum mechanics was pioneered by Couder and Fort, who pointed out the similarity between wave-driven droplets and de Broglie's picture of quantum particles propelled by "pilot-waves" [15]. The similarity led Couder and Fort to perform single- and double-slit experiments with single wave-driven droplets [15] and to contrast the double-slit experiment with the celebrated double-slit experiment in quantum mechanics [31-33]. Based on observed droplet trajectories Couder and Fort concluded that an interference pattern in the single-particle statistics is produced due to interference between surface wave components that pass the two slits while the droplet passes the one or the other slit [15]. The conclusion by Couder and Fort has been questioned in subsequent studies [26,27], and recently Pucci, Harris, Faria, and Bush have determined the single-particle statistics for both single and double slits in a refined experiment and demonstrated that the statistics are qualitatively different from the Fraunhofer diffraction and interference expressions suggested by Couder and Fort [28]. Moreover, quantitative observations of surface wave fields in slit experiments remain to be reported.

In this paper, we present an experimental study of surface wave fields in single- and double-slit experiments with single wave-driven droplets. The main purpose of our work is to characterize the wave components that pass through the two slits in the double-slit experiment and to explore the interference hypothesis stated by Couder and Fort [15]: "It can be noted that a given droplet is observed to go through one or the other of the slits. However its associated wave passes through

\footnotetext{
*Present address: Faculty of Physics, University of Vienna, Boltzmanngasse 5, Vienna 1090, Austria.

†Corresponding author: aanders@fysik.dtu.dk
} 

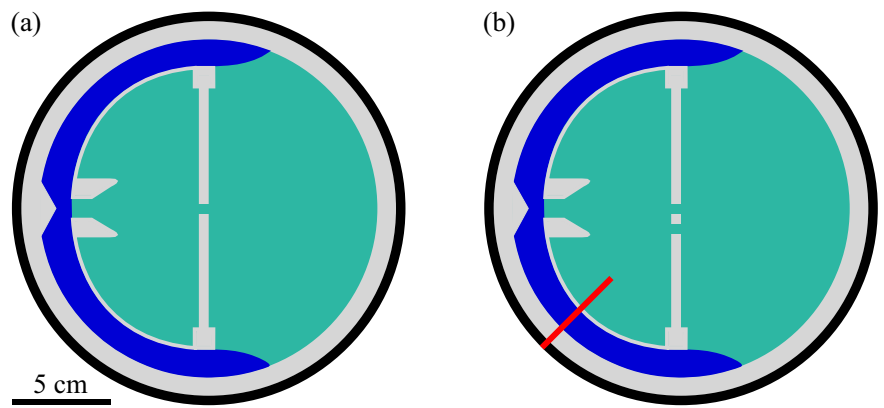

(c)

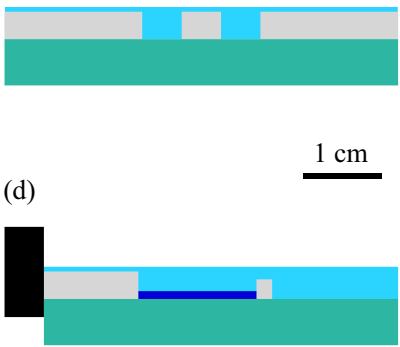

FIG. 1. Schematic illustrations of the cell geometries with narrow slits seen from above and in vertical cross-sections. (a) Single-slit, (b) double-slit, (c) vertical cross-section in the length direction of the double-slit, and (d) vertical cross-section along the red line in panel (b). The container rim (black, inner diameter $200.0 \mathrm{~mm}$ ) encloses the silicone oil bath that consists of deep areas where the surface waves can be excited and the wave-driven droplets move (green, depth $4.10 \pm 0.03 \mathrm{~mm}$ and dark blue, depth $3.1 \pm 0.1 \mathrm{~mm}$ ) and shallow areas over subsurface structures (gray, depth $0.6 \pm 0.1 \mathrm{~mm}$ ). In the dark blue region the wave-driven droplets are guided toward a launcher that aims at the single slit or the "upper" slit in the double-slit configuration. The liquid layer is indicated in light blue in the vertical cross-sections in panels (c) and (d).

both slits and the interference of the resulting waves is responsible for the trajectory of the walker." Despite their equal significance, wave fields have received little attention in comparison with the extensive studies of trajectories of wave-driven droplets. A few studies have been made of wave fields due to droplets over flat topography [5,7,8], near a straight subsurface barrier [9-11], and near a circular subsurface pillar $[10,13]$. However, only trajectories and qualitative wave field images have been reported in single- and double-slit experiments [11,15,26,28]. Quantitative exploration of wave fields in complex topography is therefore crucially needed to develop models of diffraction of wave-driven droplets.

\section{EXPERIMENTS}

Throughout our experiments, we used a circular cell with interchangeable insets (Fig. 1). The bottom of the cell consisted of a circular disk of glass that was sandwiched at its rim between a lower and an upper aluminum ring. The cell was connected firmly by an ABS plastic cone to the table of a Brüel \& Kjær 4808 vibration exciter that was leveled and mounted on a heavy optical breadboard. The cell was forced vertically with simple harmonic motion that was controlled by means of a feedback system consisting of a Spider 81B box and a Deltatron 4533-B-001 accelerometer mounted vertically on the lower aluminum ring. It is important to ensure uniformity of the vertical acceleration and to avoid horizontal accelerations of the cell [34]. With typical working parameters, we found a $1 \%$ variation in the vertical acceleration and horizontal acceleration with amplitude of less than $2 \%$ of the vertical acceleration amplitude. During the runs, a transparent lid of PMMA plastic was mounted with screws on top of the container rim to avoid disturbances due to ambient air currents.

Different single- and double-slit configurations were established by placing insets with subsurface barriers of aluminum on the glass bottom (Fig. 1). The subsurface barriers were $3.5 \pm 0.1 \mathrm{~mm}$ in height and $5.0 \pm 0.1 \mathrm{~mm}$ in horizontal thickness. We carried out the experiments using narrow slits with widths $w=5.7 \pm 0.1 \mathrm{~mm}$ and wide slits with widths $w=15.0 \pm 0.1 \mathrm{~mm}$, respectively. The central subsurface barriers in the double-slit configurations were $5.0 \pm 0.1 \mathrm{~mm}$ wide, and the corresponding distances between the slit centers in the double-slit experiments were therefore $d=10.7 \pm 0.1 \mathrm{~mm}$ and $d=20.0 \pm 0.1 \mathrm{~mm}$, respectively. The filling depth of the cell was $4.10 \pm 0.03 \mathrm{~mm}$ in all experiments. 
We used a silicone oil with kinematic viscosity $20 \mathrm{cSt}$, density $0.95 \mathrm{~g} / \mathrm{mL}$, surface tension $21 \mathrm{mN} / \mathrm{m}$, and index of refraction 1.40 at $25^{\circ} \mathrm{C}$. The experiments with narrow and wide slits, respectively, were carried out using silicone oil from two different $1 \mathrm{~L}$ bottles (Sigma Aldrich). Droplets with well-controlled diameter $D$ were made using a droplet-on-demand generator [35]. The forcing frequency was $80 \mathrm{~Hz}$ and a representative forcing amplitude was $\gamma=4.16 \pm 0.01 \mathrm{~g}$, where $g$ is the acceleration due to gravity. The Faraday wavelength was $4.75 \mathrm{~mm}$ in the deep areas. We estimated the uncertainty in the forcing amplitude from its variation in uniformity over the central $60 \times 60 \mathrm{~mm}^{2}$ area. We checked the spatial uniformity of the acceleration amplitude threshold for Faraday surface waves $\gamma_{\mathrm{F}}$, and we measured calibration curves between $\gamma_{\mathrm{F}}$ and the surface temperature using a Fluke 574 infrared thermometer and visual inspection of the surface. We determined $\gamma_{\mathrm{F}}$ using our calibration curves to take into account the slow $1^{\circ} \mathrm{C}$ temperature increase in each run sequence. The full temperature range for all run sequences was $21.5-24.3^{\circ} \mathrm{C}$, and we estimated the uncertainty in temperature to be $\pm 0.1{ }^{\circ} \mathrm{C}$ in each run. A representative threshold amplitude for Faraday surface waves was $\gamma_{\mathrm{F}}=4.22 \pm 0.01 \mathrm{~g}$.

The proximity to the threshold for Faraday surface waves is in the literature described by the amplitude ratio, $\alpha=\gamma / \gamma_{\mathrm{F}}$, or the memory parameter, $\Gamma^{-1}=\gamma_{\mathrm{F}} /\left(\gamma_{\mathrm{F}}-\gamma\right)$, that provides a rough estimate of the number of earlier droplet impacts that contribute to the wave field $[5,7,28]$. The relative uncertainty $\sigma_{\Gamma^{-1}} / \Gamma^{-1}$ follows from propagation of errors, and it is proportional to $\Gamma^{-1}$ itself

$$
\frac{\sigma_{\Gamma^{-1}}}{\Gamma^{-1}} \approx \Gamma^{-1} \sqrt{\left(\frac{\sigma_{\gamma}}{\gamma}\right)^{2}+\left(\frac{\sigma_{\gamma_{\mathrm{F}}}}{\gamma_{\mathrm{F}}}\right)^{2}},
$$

where we have assumed that the relative uncertainties $\sigma_{\gamma} / \gamma$ and $\sigma_{\gamma_{\mathrm{F}}} / \gamma_{\mathrm{F}}$ are uncorrelated. Even small relative uncertainties in $\gamma$ and $\gamma_{\mathrm{F}}$ can therefore lead to large relative uncertainty in $\Gamma^{-1}$. The relative uncertainty $\sigma_{\Gamma^{-1}} / \Gamma^{-1}$ should be less than unity to ensure that we are always working below the Faraday threshold. We find $\Gamma^{-1}=70 \pm 16$ in our representative example, and $\Gamma^{-1} \approx 50-100$ is an upper limit on the memory parameter in our experiments. We note that

$$
\frac{\sigma_{\alpha}}{1-\alpha}=\frac{\sigma_{\Gamma^{-1}}}{\Gamma^{-1}}
$$

and our requirement, $\sigma_{\Gamma^{-1}} / \Gamma^{-1}<1$, is therefore equivalent to the requirement $\sigma_{\alpha}<1-\alpha$, which should be considered if $\alpha$ is reported instead of $\Gamma^{-1}$.

We determined the instantaneous surface wave fields using the free-surface synthetic Schlieren technique $[5,10,16,36]$. The technique relies on the refraction of a random dot-pattern that is observed through the surface. The height gradient field is determined from the observed displacement field of the dot-pattern relative to its reference image, and subsequently the height field is obtained by integration [37]. An $80 \times 100 \mathrm{~mm}^{2}$ area in the central part of the cell was observed from above using a digital video camera (Mako U-135B, spatial resolution $1024 \times 1280$ pixel) that was positioned $190 \mathrm{~cm}$ above the silicone oil surface. The videos were made at the Faraday wave frequency $40 \mathrm{~Hz}$ with exposure time $170 \mu \mathrm{s}$. We used the vibration exciter drive signal to trigger the recordings at the maximum height of the central wave crest corresponding to zero vertical displacement and downward motion of the cell. In the reconstruction procedure, we used $16 \times 16$ pixel interrogation windows with $75 \%$ overlap resulting in a height field with $0.31 \times 0.31 \mathrm{~mm}^{2}$ spatial resolution. The displacement field determination was carried out using the open source software PIVlab 2.02 [38]. To correct for a possible mean translation we subtracted the mean displacement from the displacement field [36], we found only a negligible mean rotation [10], and we subsequently applied a band-pass filter centered at the Faraday wavelength. We validated the technique using known optical glass components that were placed in the empty cell, and we estimated the uncertainty to be $\pm 3 \mu \mathrm{m}$. 

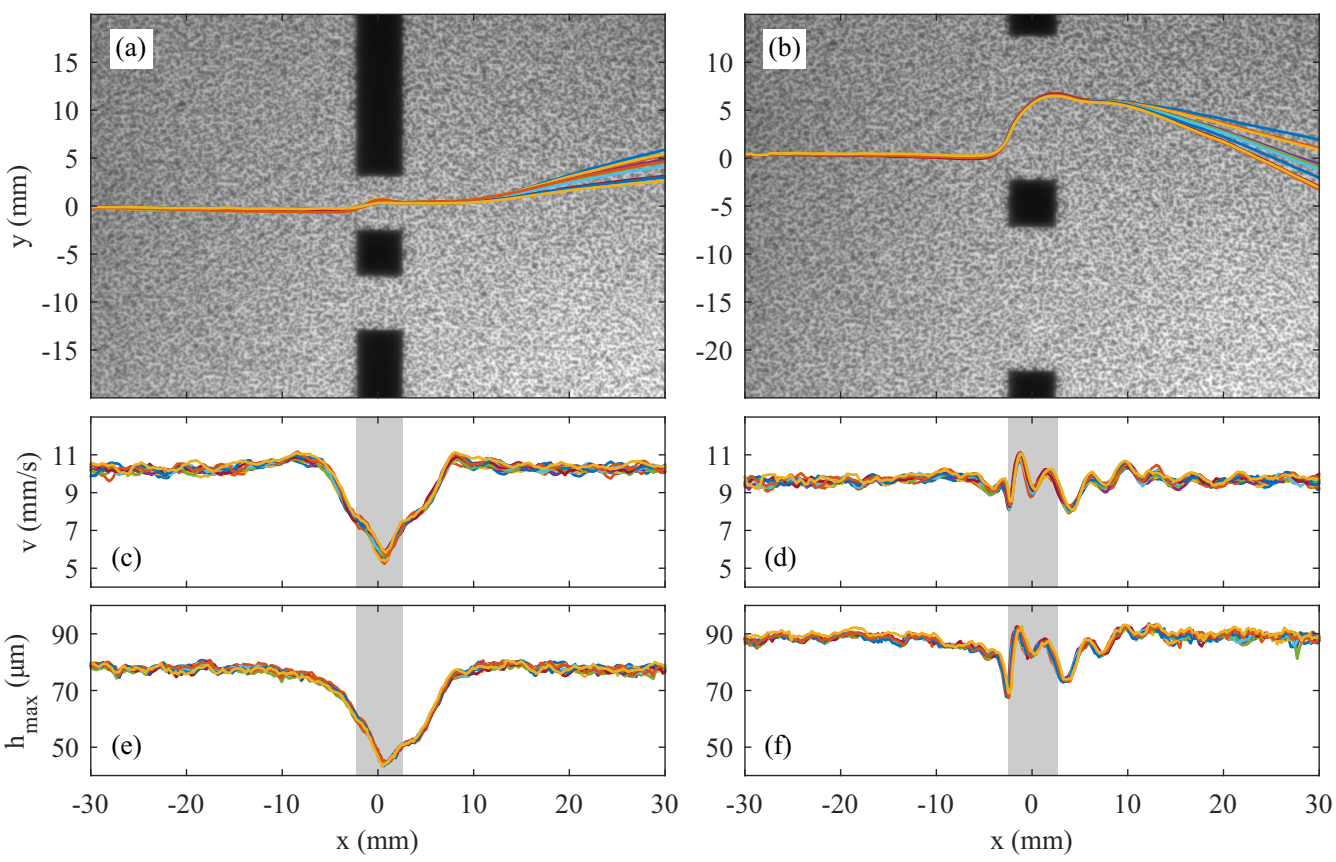

FIG. 2. Droplet trajectories, speeds, and central wave crest heights in the double-slit experiments. (a), (b) Ten representative trajectories for narrow and wide slits, respectively, with the droplets moving from left to right. The top views of the trajectories are superimposed on the reference images of the dot-pattern, and the subsurface barriers appear black. (c), (d) The corresponding speeds, and (e), (f) the corresponding central wave crest heights. The narrow slit width was $w=5.7 \pm 0.1 \mathrm{~mm}$, the distance between the slit centers $d=10.7 \pm$ $0.1 \mathrm{~mm}$, the droplet diameter $D=767 \pm 2 \mu \mathrm{m}$, and the memory parameters for the different trajectories were in the range $64<\Gamma^{-1}<83$. The wide slit width was $w=15.0 \pm 0.1 \mathrm{~mm}$, the distance between the slit centers $d=20.0 \pm 0.1 \mathrm{~mm}$, the droplet diameter $D=766 \pm 3 \mu \mathrm{m}$, and the memory parameters for the different trajectories were in the range $67<\Gamma^{-1}<107$.

\section{RESULTS}

We explored four different configurations, i.e., single- and double-slit experiments with narrow and wide slits, respectively. For each configuration we recorded approximately ten droplet passages with the same impact parameters. The droplet launcher directed the droplets with normal incidence toward the slit arrangements as in most previous experiments [15,28]. We chose the impact parameters so that the droplets passed near the central barrier in the double-slit experiments to facilitate wave excitation in and through the slit that was not visited by the droplets. Furthermore, we worked at the highest values of the memory parameter that we could establish while remaining below the threshold for Faraday surface waves in a well-controlled manner. The higher the memory parameter is, the further the wave field extends spatially [5], and the larger we presume that the influence will be of the slit that is not visited by the droplet.

The droplet trajectories, the speeds, and the central wave crest heights in the double-slit experiments show characteristic features and serve to illustrate the degree of reproducibility (Fig. 2). The droplets were directed by the launcher toward the "upper" slit in the double-slit configuration. The droplets are deflected toward the center line when they enter the slit, and they exit from the middle half of the slit (Fig. 2). It is well-documented that wave-driven droplets over flat topography typically move with constant velocity [4,6]. Our measured trajectories are straight when the droplets are further than approximately two Faraday wavelengths from the 

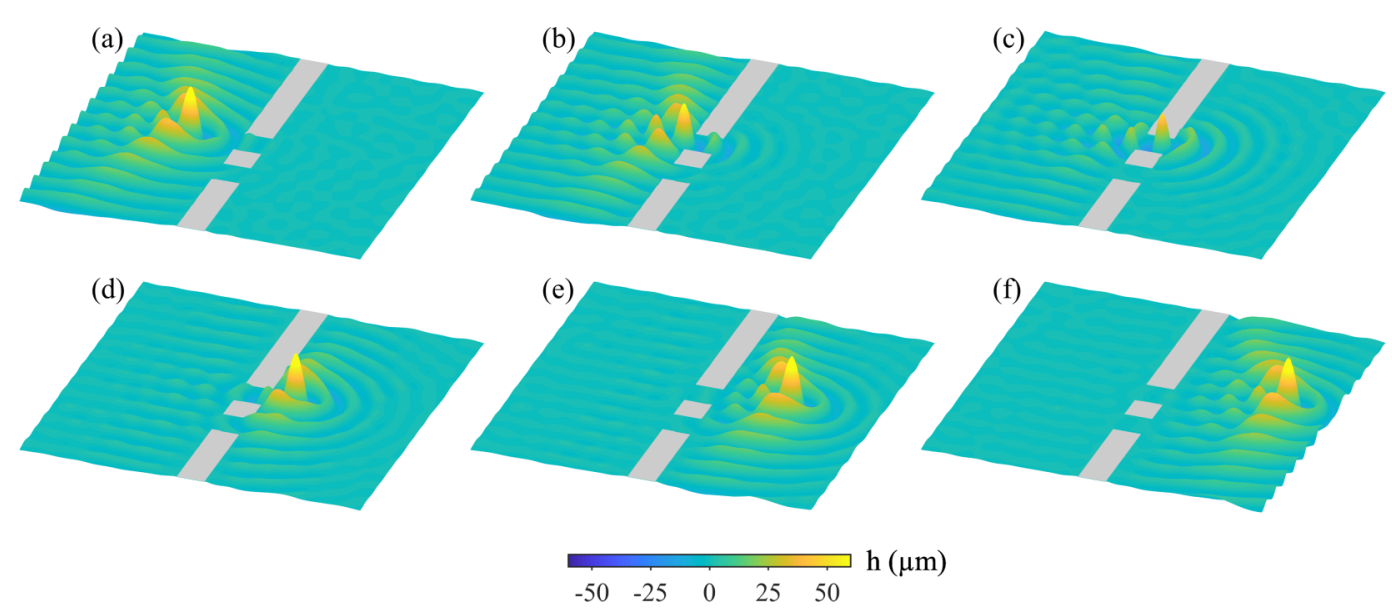

FIG. 3. Time-series of wave fields in the double-slit experiment with narrow slits and parameters $w=5.7 \pm 0.1 \mathrm{~mm}, d=10.7 \pm 0.1 \mathrm{~mm}, D=767 \pm 2 \mu \mathrm{m}$, and $\Gamma^{-1}=70 \pm 16$. The time-step between consecutive images is $0.75 \mathrm{~s}$, and the subsurface barriers are indicated in gray. (a) The wave-driven droplet is approaching the double-slit and the wave field has the horseshoe-shape that is characteristic of a droplet over flat topography. (b) The droplet is entering the slit and a slight reduction of the central wave crest is observed. (c) Overall the wave field amplitude is reduced as the droplet is leaving the slit, but in front of the droplet the wave field extends further than when the droplet is over flat topography. (d) The droplet is clear of the slit. (e) The droplet is at the position where the trajectories spread out (Fig. 2). (f) The horseshoe-shape is re-established. In all the images the wave component is weak in and through the slit that is not visited by the droplet.

subsurface barriers, and correspondingly the measured speeds are constant. The droplet speeds of $v=10.2 \pm 0.1 \mathrm{~mm} / \mathrm{s}$ [Fig. 2(c)] and $v=9.6 \pm 0.1 \mathrm{~mm} / \mathrm{s}$ [Fig. 2(d)] are in good agreement with previous work [4,6]. The corresponding central wave crest heights are $h_{\max }=78 \pm 3 \mu \mathrm{m}$ [Fig. 2(e)] and $h_{\max }=89 \pm 3 \mu \mathrm{m}$ [Fig. 2(f)]. We presume that the differences in droplet speeds and central wave crest heights are due to differences in the properties of the silicone oil from the two different $1 \mathrm{~L}$ bottles used for the experiments with narrow and wide slits, respectively. During the passage of the narrow slits the droplet speed is reduced by a factor of two [26], whereas the wide slits lead to a much less pronounced speed reduction during slit passage. The droplet speed and the height of the central wave crest during slit passage are closely correlated for both the narrow slits and the wide slits. The variability in the trajectories is similar to the variability in the previously reported single-slit trajectories with a comparable value of the memory parameter [28]. Characteristically the droplet trajectories follow each other closely until the local maximum in speed after slit passage is reached, and subsequently they are deflected with some spread in deflection angle. The cause of this characteristic behavior remains to be explained.

To illustrate the wave fields during slit passage we present measured time-series of wave fields in the single- and double-slit experiments (Fig. 3 and Supplemental Material, movies 1-4 [39]). When the wave-driven droplet is approaching the double-slit, the wave field has the horseshoe-shape that is characteristic of a droplet over flat topography (Fig. 3). Superposition of the wave field contributions from the earlier droplet impacts leads to an interference pattern with intense, transversal "wings" on both sides of the droplet trajectory $[5,7,8]$. The wing structures are easily visible up to five Faraday wavelengths from the droplet. The wave component in and through the slit that is not visited by the droplet is weak in the double-slit experiment with narrow slits (Fig. 3). In contrast, the wave excitation in the slit that is visited by the droplet leads to a strong wave component up to four Faraday wavelengths in front of the droplet. We presume that this wave amplification underlies the local maximum in droplet speed after slit passage and the subsequent spread in deflection angle (Fig. 2). 

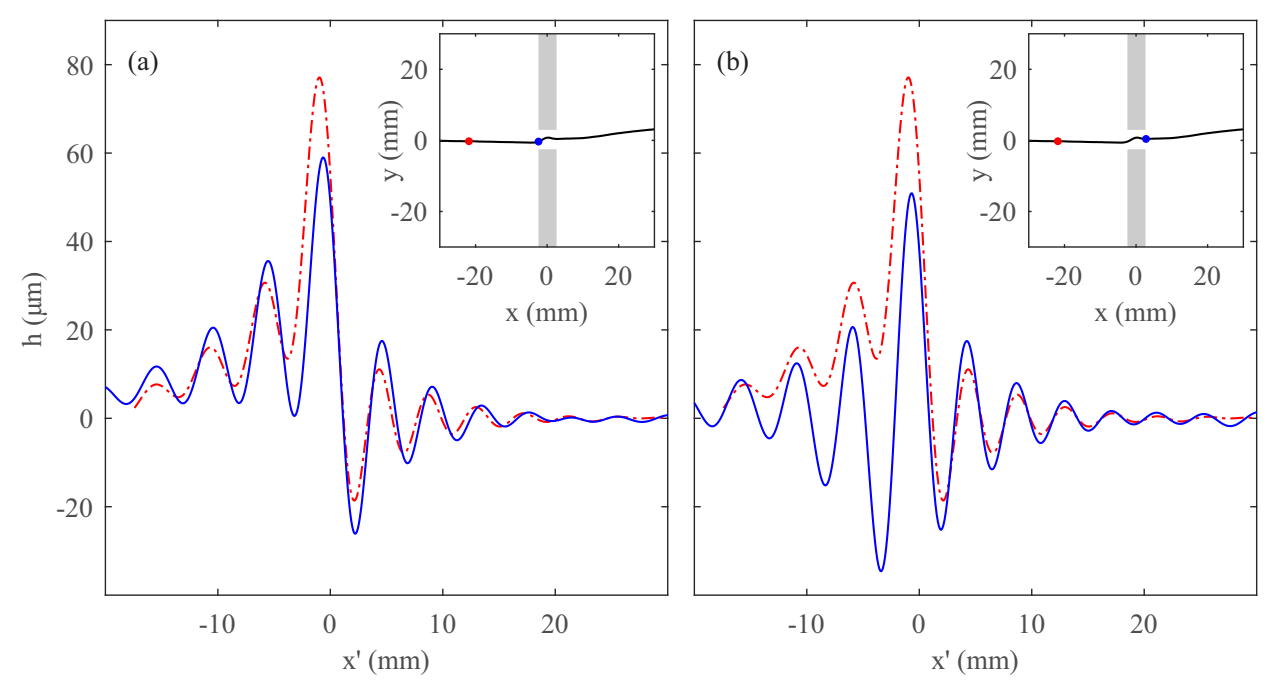

FIG. 4. The wave heights when the droplet is far from the subsurface barriers, and when it is in the slit in the single-slit experiment with a narrow slit and parameters $w=5.7 \pm 0.1 \mathrm{~mm}, D=763 \pm 3 \mu \mathrm{m}$, and $\Gamma^{-1}=69 \pm 16$. The insets show the droplet trajectory (solid line, black) and the subsurface barriers (gray). Both panels show the wave height (dash-dot line, red) when the droplet is at the position indicated in the insets by the red dot. (a) The wave height (solid line, blue) when the droplet is entering the slit (inset, blue dot), and (b) the wave height (solid line, blue) when the droplet is leaving the slit (inset, blue dot). The instantaneous surface heights are determined along the straight lines with constant $y$ coordinate through the droplet positions, and they are shown as functions of the $x$ coordinate relative to the $x$ coordinate of the droplet.

The characteristics of the wave field during slit passage can be quantified by comparing the wave heights when the droplet is in the slit opening in the single-slit experiment with a narrow slit and the wave heights when the droplet is over flat topography and far from the subsurface barriers (Fig. 4). We observe a slight reduction of the central wave crest and an amplification of the wave crests in front of the droplet when it is entering the slit [Fig. 4(a)]. When the droplet is leaving the slit, we observe a reduction of the central wave crest, an alteration of the wave field behind the droplet, and an amplification of the wave crests in front of it [Fig. 4(b)]. Similar characteristics can presumably be found with wave-driven droplets that move in linear channels [12].

To explore the interference hypothesis we contrast droplet trajectories and wave fields in the single- and double-slit experiments (Fig. 5). In the narrow configurations we find that the trajectories (white curves) with the selected impact parameters are similar and within the range of variability observed for each individual configuration (Fig. 5, left panels). The wave component in and through the slit that is not visited by the droplet is weak, and the wave fields around the droplets are similar in the two situations as shown by comparison of the wave fields when the droplet enters and leaves the slit, respectively. A qualitatively similar scenario is found for the wide configurations where we observe that the central barrier in the double-slit experiment shadows and hinders wave component excitation in the slit that is not visited by the droplet (Fig. 5, right panels).

To quantitatively compare the wave fields in the single- and double-slit experiments we display the wave heights along the center line of the slit that is visited by the droplet (Fig. 6). To test the interference hypothesis we focus on the wave heights when the droplets are entering the slit opening, and we show the wave heights along the center line since this is where the droplets move when exiting the slit opening. With both narrow and wide slits we find minute differences in the wave heights on the order of a few micrometer, and the differences are comparable with the differences between different realizations in the individual configurations. 

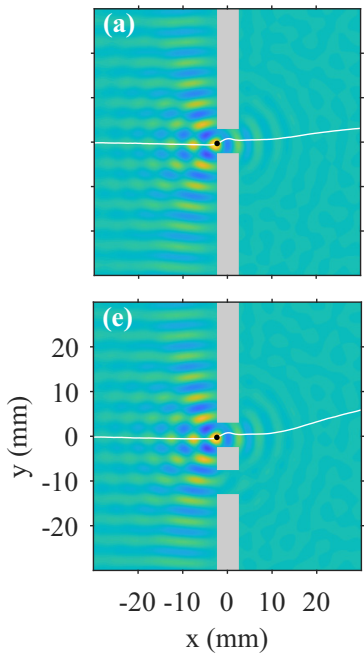
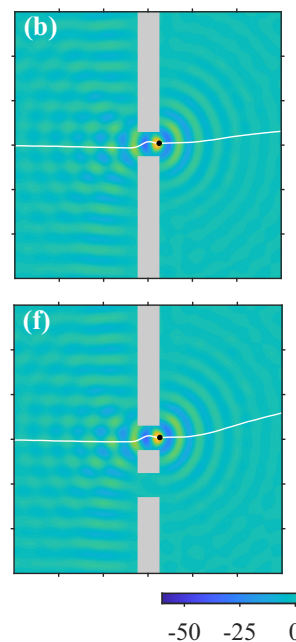
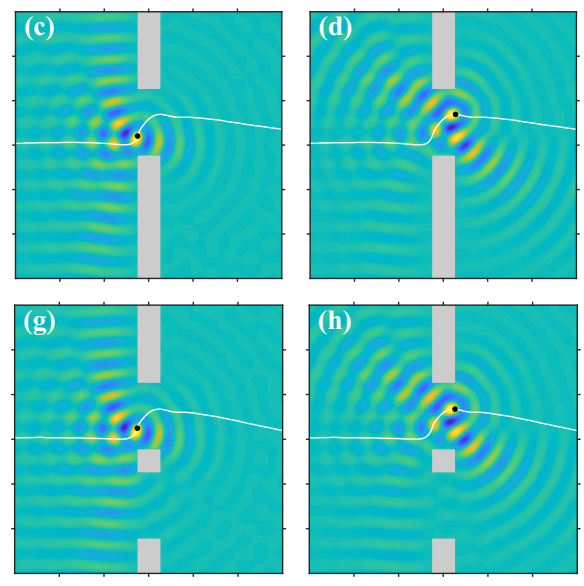

FIG. 5. Wave fields in single- and double-slit experiments with slits of different widths when the droplet enters and leaves the slit, respectively. Droplet trajectories (white curves) and instantaneous droplet positions (black dots). (a, b) Single-slit experiment with a narrow slit $w=5.7 \pm 0.1 \mathrm{~mm}, D=763 \pm 3 \mu \mathrm{m}$, and $\Gamma^{-1}=$ $69 \pm 16$, (c, d) single-slit experiment with a wide slit $w=15.0 \pm 0.1 \mathrm{~mm}, D=768 \pm 2 \mu \mathrm{m}$, and $\Gamma^{-1}=$ $71 \pm 16$, (e, f) double-slit experiment with narrow slits $w=5.7 \pm 0.1 \mathrm{~mm}, d=10.7 \pm 0.1 \mathrm{~mm}, D=$ $767 \pm 2 \mu \mathrm{m}$, and $\Gamma^{-1}=70 \pm 16$, and $(\mathrm{g}$, h) double-slit experiment with wide slits $w=15.0 \pm 0.1 \mathrm{~mm}, d=$ $20.0 \pm 0.1 \mathrm{~mm}, D=766 \pm 3 \mu \mathrm{m}$, and $\Gamma^{-1}=67 \pm 14$. The wave component in and through the slit that is not visited by the droplet is weak in both double-slit experiments, and there are no evident differences between the wave fields in the comparable single- and double-slit experiments.
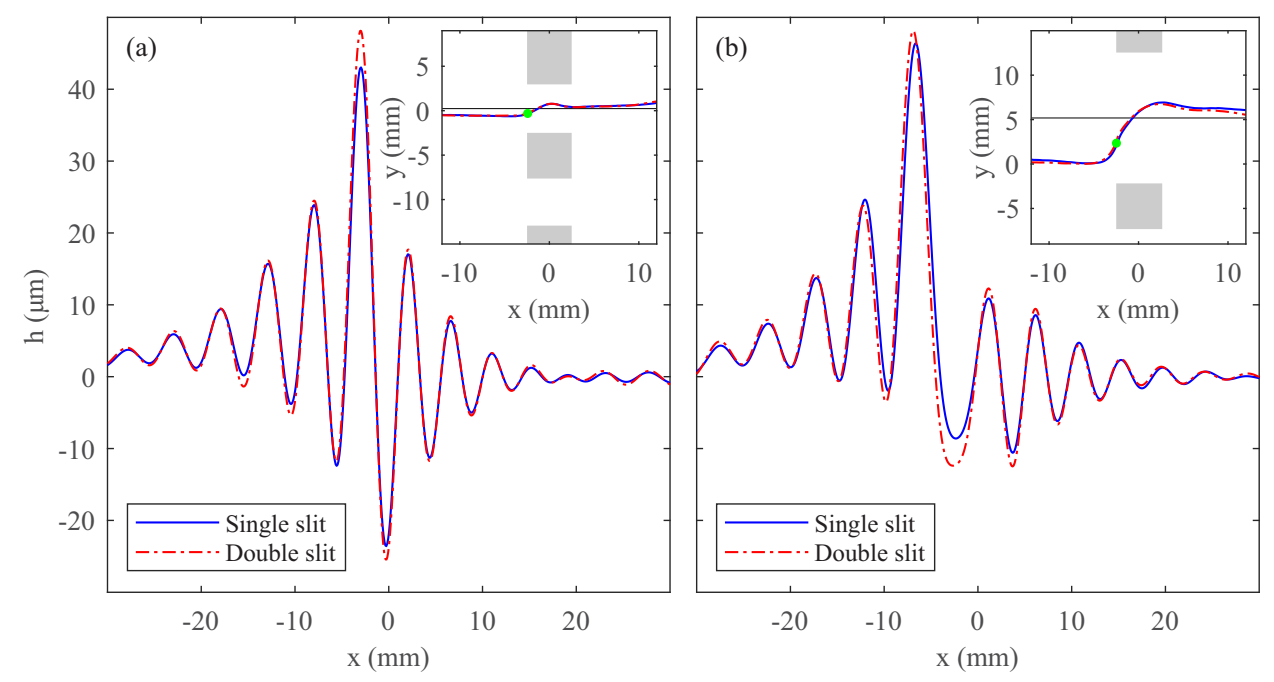

FIG. 6. Comparison of the wave heights along the center line of the slit that is visited by the droplet when the droplet enters the slit in the single- and double-slit experiment, respectively. (a) Narrow slits and (b) wide slits. The wave heights are shown for the single slits (solid lines, blue) and the double slits (dash-dot lines, red). The four trajectories are identical to those displayed in Fig. 5. The insets show top views of the single-slit trajectories (solid lines, blue), the double-slit trajectories (dash-dot lines, red), the droplet locations (green dots), the double-slit arrangements (gray), and the center lines (solid lines, black). 


\section{DISCUSSION AND OUTLOOK}

Both droplet trajectories and wave fields are affected significantly during slit passage, and in the single-slit configurations this leads to interesting droplet diffraction patterns $[15,28]$. However, droplet trajectory and wave field are predominantly influenced by the slit that is visited by the droplet, and in the double-slit experiments the wave component in and through the slit that is not visited by the droplet is weak (Fig. 5). Furthermore, the wave heights along the center line in the slit that is visited by the droplet show minute differences between the comparable single- and double-slit configurations regardless of the slit width (Fig. 6). These observations cast doubt on the interference hypothesis put forward by Couder and Fort in their double-slit experiment [15].

The central barrier in the double-slit experiments hinders wave component excitation in the slit that is not visited by the droplet even in the wide configuration. We expect that at higher memory the wave field will also appear with the characteristic horseshoe-shape, and that wave field excitation will not take place through the slit that is not visited by the droplet when it moves with normal incidence. Our findings do not exclude different trajectories and single-particle statistics in comparable single- and double-slit experiments due to the slit that is not visited by the droplet, since wave field formation and structure on the droplet side of the slit arrangement can be influenced by the differences in boundary conditions [26]. Such effects can possibly account for the observed differences between the droplet trajectories in the comparable single- and double-slit experiments by Pucci, Harris, Faria, and Bush [28, Fig. 8].

We have focused on a particular choice of silicone oil, droplet diameter, liquid depth, slit geometries, and impact parameters, and we have worked with the highest value of the memory parameter that is possible with our degree of experimental control. It could be interesting to explore trajectories and wave fields in other experimental realizations, in particular at high values of the memory parameter at which it has been suggested that the droplet trajectories are chaotic [28]. Furthermore, we hope that theoretical models of diffraction of wave-driven droplets will be able to shed light on our presumptions, and that our wave field observations will help development and testing of such models.

\section{ACKNOWLEDGMENTS}

We are thankful to D. M. Harris for fruitful discussions of experimental design, T. Bohr, M. T. Levinsen, and A. Duchesne for helpful comments and suggestions, and W. Qiu for assistance with index of refraction measurements. We also thank E. Hansen for his careful work in the design and construction of the experimental setup. We gratefully acknowledge funding from the Carlsberg Foundation through Grant No. 2013_01_0951.

[1] Y. Couder, E. Fort, C.-H. Gautier, and A. Boudaoud, From Bouncing to Floating: Noncoalescence of Drops on a Fluid Bath, Phys. Rev. Lett. 94, 177801 (2005).

[2] Y. Couder, S. Protière, E. Fort, and A. Boudaoud, Walking and orbiting droplets, Nature 437, 208 (2005).

[3] S. Protière, Y. Couder, E. Fort, and A. Boudaoud, The self-organization of capillary wave sources, J. Phys.: Condens. Matter 17, S3529 (2005).

[4] S. Protière, A. Boudaoud, and Y. Couder, Particle-wave association on a fluid interface, J. Fluid Mech. 554, 85 (2006).

[5] A. Eddi, E. Sultan, J. Moukhtar, E. Fort, M. Rossi, and Y. Couder, Information stored in Faraday waves: The origin of a path memory, J. Fluid Mech. 674, 433 (2011).

[6] J. Moláček and J. W. M. Bush, Drops walking on a vibrating bath: Towards a hydrodynamic pilot-wave theory, J. Fluid Mech. 727, 612 (2013).

[7] A. U. Oza, R. R. Rosales, and J. W. M. Bush, A trajectory equation for walking droplets: Hydrodynamic pilot-wave theory, J. Fluid Mech. 737, 552 (2013). 
[8] P. A. Milewski, C. A. Galeano-Rios, A. Nachbin, and J. W. M. Bush, Faraday pilot-wave dynamics: Modelling and computation, J. Fluid Mech. 778, 361 (2015).

[9] G. Pucci, P. J. Sáenz, L. M. Faria, and J. W. M. Bush, Non-specular reflection of walking droplets, J. Fluid Mech. 804, R3 (2016).

[10] A. P. Damiano, P.-T. Brun, D. M. Harris, C. A. Galeano-Rios, and J. W. M. Bush, Surface topography measurements of the bouncing droplet experiment, Exp. Fluids 57, 163 (2016).

[11] L. M. Faria, A model for Faraday pilot waves over variable topography, J. Fluid Mech. 811, 51 (2017).

[12] B. Filoux, M. Hubert, P. Schlagheck, and N. Vandewalle, Walking droplets in linear channels, Phys. Rev. Fluids 2, 013601 (2017).

[13] D. M. Harris, P.-T. Brun, A. Damiano, L. M. Faria, and J. W. M. Bush, The interaction of a walking droplet and a submerged pillar: From scattering to the logarithmic spiral, Chaos 28, 096105 (2018).

[14] R. N. Valani, A. C. Slim, and T. Simula, Superwalking Droplets, Phys. Rev. Lett. 123, 024503 (2019).

[15] Y. Couder and E. Fort, Single-Particle Diffraction and Interference at a Macroscopic Scale, Phys. Rev. Lett. 97, 154101 (2006).

[16] A. Eddi, E. Fort, F. Moisy, and Y. Couder, Unpredictable Tunneling of a Classical Wave-Particle Association, Phys. Rev. Lett. 102, 240401 (2009).

[17] E. Fort, A. Eddi, A. Boudaoud, J. Moukhtar, and Y. Couder, Path-memory induced quantization of classical orbits, Proc. Natl. Acad. Sci. USA 107, 17515 (2010).

[18] J. W. M. Bush, Quantum mechanics writ large, Proc. Natl. Acad. Sci. USA 107, 17455 (2010).

[19] A. Eddi, J. Moukhtar, S. Perrard, E. Fort, and Y. Couder, Level Splitting at Macroscopic Scale, Phys. Rev. Lett. 108, 264503 (2012).

[20] D. M. Harris, J. Moukhtar, E. Fort, Y. Couder, and J. W. M. Bush, Wavelike statistics from pilot-wave dynamics in a circular corral, Phys. Rev. E 88, 011001(R) (2013).

[21] D. M. Harris and J. W. M. Bush, Droplets walking in a rotating frame: From quantized orbits to multimodal statistics, J. Fluid Mech. 739, 444 (2014).

[22] A. U. Oza, D. M. Harris, R. R. Rosales, and J. W. M. Bush, Pilot-wave dynamics in a rotating frame: On the emergence of orbital quantization, J. Fluid Mech. 744, 404 (2014).

[23] S. Perrard, M. Labousse, M. Miskin, E. Fort, and Y. Couder, Self-organization into quantized eigenstates of a classical wave-driven particle, Nat. Commun. 5, 3219 (2014).

[24] S. Perrard, M. Labousse, E. Fort, and Y. Couder, Chaos Driven by Interfering Memory, Phys. Rev. Lett. 113, 104101 (2014).

[25] J. W. M. Bush, Pilot-Wave Hydrodynamics, Annu. Rev. Fluid Mech. 47, 269 (2015).

[26] A. Andersen, J. Madsen, C. Reichelt, S. Rosenlund Ahl, B. Lautrup, C. Ellegaard, M. T. Levinsen, and T. Bohr, Double-slit experiment with single wave-driven particles and its relation to quantum mechanics, Phys. Rev. E 92, 013006 (2015).

[27] T. Bohr, A. Andersen, and B. Lautrup, Bouncing Droplets, Pilot-Waves, and Quantum Mechanics, in Recent Advances in Fluid Dynamics with Environmental Applications, edited by J. Klapp, L. D. G. Sigalotti, A. Medina, A. López, and G. Ruiz-Chavarría (Springer International Publishing, Berlin, 2016), pp. 335-349.

[28] G. Pucci, D. M. Harris, L. M. Faria, and J. W. M. Bush, Walking droplets interacting with single and double slits, J. Fluid Mech. 835, 1136 (2018).

[29] P. J. Sáenz, T. Cristea-Platon, and J. W. M. Bush, Statistical projection effects in a hydrodynamic pilotwave system, Nat. Phys. 14, 315 (2018).

[30] T. Bohr, Quantum physics dropwise, Nat. Phys. 14, 209 (2018).

[31] R. P. Feynman, R. B. Leighton, and M. Sands, The Feynman Lectures on Physics (Addison-Wesley, Boston, MA, 1965).

[32] A. Tonomura, J. Endo, T. Matsuda, and T. Kawasaki, Demonstration of single-electron buildup of an interference pattern, Am. J. Phys. 57, 117 (1989).

[33] R. Bach, D. Pope, S.-H. Liou, and H. Batelaan, Controlled double-slit electron diffraction, New J. Phys. 15, 033018 (2013).

[34] D. M. Harris and J. W. M. Bush, Generating uniaxial vibration with an electrodynamic shaker and external air bearing, J. Sound Vib. 334, 255 (2015). 
[35] D. M. Harris, T. Liu, and J. W. M. Bush, A low-cost, precise piezoelectric droplet-on-demand generator, Exp. Fluids 56, 83 (2015).

[36] F. Moisy, M. Rabaud, and K. Salsac, A synthetic Schlieren method for the measurement of the topography of a liquid interface, Exp. Fluids 46, 1021 (2009).

[37] A. Agrawal, R. Raskar, and R. Chellappa, What is the Range of Surface Reconstructions from a Gradient Field?, in Proceedings of the ECCV 2006, Part I, edited by A. Leonardis, H. Bischof, and A. Pinz (Springer-Verlag, Berlin, 2006), pp. 578-591.

[38] W. Thielicke and E. J. Stamhuis, PIVlab-Towards User-friendly, Affordable, and Accurate Digital Particle Image Velocimetry in MATLAB, J. Open Res. Software 2, e30 (2014).

[39] See Supplemental Material at http://link.aps.org/supplemental/10.1103/PhysRevFluids.4.104801 for movies showing measured wave fields in single- and double-slit experiments. 